\title{
Spectral linewidth in microcavity surface-emitting lasers
}

\author{
Igor Vurgaftman and Jasprit Singh \\ Solid State Electronics Laboratory, Department of Electrical Engineering and Computer Science, \\ The University of Michigan, Ann Arbor, Michigan 48109-2122
}

(Received 13 May 1994; accepted for publication 29 July 1994)

The Schawlow-Townes expression for the laser linewidth predicts a substantial $\mathrm{cw}$ linewidth enhancement in microcavity lasers, in which a large fraction of spontaneous emission is directed into the lasing mode, in contrast with conventional semiconductor lasers, in which the lasing mode accepts only a tiny fraction of spontaneously emitted photons. By performing a theoretical analysis of rigorous solutions of the wave equation in the surface-emitting cavity and of the band structure in the active region, it is shown that the increase in the linewidth is much slower than the increase in the spontaneous-emission factor $\beta$ because of reductions in the total spontaneous emission rate, the threshold carrier density, and the linewidth enhancement factor and an increased slope of the light-current characteristic obtained for the microcavity laser in steady state. Also much smaller driving currents in a microcavity laser are required to achieve the same power output compared with conventional semiconductor lasers, resulting in a linewidth of the order of several hundred $\mathrm{MHz}$ at moderate driving currents for the former. We also discuss the various factors influencing the linewidth in microcavity and strained quantum well lasers and the relation between lasing threshold and spectral linewidth in both macroscopic and microscopic cavity lasers.

\section{INTRODUCTION}

The availability of single-mode semiconductor lasers made initial precise measurements of the $\mathrm{cw}$ spectral width of single-mode emission in laser diodes possible. ${ }^{1}$ Interestingly, the results indicated a factor-of-50 discrepancy with theoretical predictions based on the standard SchawlowTownes expression. ${ }^{2}$ In order to explain the experimental results, the theory of the linewidth in semiconductor lasers was reexamined. ${ }^{3}$ It was shown that additional linewidth broadening arises from retarded amplitude fluctuations caused by spontaneous emission induced refractive index variations. These were quantified in terms of the linewidth enhancement factor $\alpha$ equal to the ratio of the real and imaginary parts of the differential susceptibility, ${ }^{4}$ measurements of which ${ }^{5}$ produced a theoretically expected value close to 5 . Calculations of the linewidth enhancement factor in quantum well lasers indicated a factor of 2-3 reduction ${ }^{6}$ due to the decreased asymmetry of the differential gain around the lasing energy. Many refinements of the original formalism have been proposed in order to account for the power-independent contribution $^{7}$ and deviations from the inverse power proportionality. ${ }^{8}$ Recently, the semiconductor laser linewidth has been calculated using a nonequilibrium Green's-function formalism. The amount of linewidth broadening in excess of that predicted by the Schawlow-Townes expression has been attributed to the effects of gain saturation, ${ }^{10}$ currently thought to contribute only a small broadening. ${ }^{11}$ Successful application of the currently accepted formalism to new types of semiconductor lasers with quantum confinement of carriers and photons is likely to serve as a test of its validity.

Microcavity lasers have attracted considerable interest of semiconductor laser researchers of late that has been fueled by the possibility of drastic threshold reduction by means of modifying the optical-mode density using a laser cavity with all dimensions of the order of the photon wavelength. ${ }^{12}$ The extent of spontaneous emission factor increas $\mathrm{e}^{13}$ and threshold reduction ${ }^{14,15}$ has been examined for the surface-emitting laser geometry. The efficiency of microcavity lasers is owed to the fact that a large fraction $(>0.1)$ of spontaneously emitted photons is directed into the lasing mode compared with a tiny fraction $\left(\approx 10^{-5}\right)$ in conventional cavity laser diodes. When the fraction reaches unity the lasing threshold transition disappears as the distinction between spontaneous and stimulated emission becomes less relevant. The enhancement of the spontaneous emission factor may be explained by the formation of a resonance in the photon density of states close to the interband transitions in the active medium dictated by the boundary conditions in the cavity. Although both static and dynamic characteristics of microcavity surface-emitting lasers with practically realizable dimensions have been studied, ${ }^{15}$ the issue of the linewidth in microcavity lasers has received relatively little attention. In this paper, a comparative study of microcavity surface-emitting lasers with strained quantum well active regions and quantum well and bulk DFB lasers is presented using a modification of the Schawlow-Townes formula with the linewidth enhancement factor based on the Langevin approach. ${ }^{16}$

\section{FORMALISM}

The modified Schawlow-Townes formula as it is usually applied in theoretical fits of the linewidth measurements is couched in terms of the linewidth of the passive resonator and contains two parameters whose precise values are not directly measurable: the inversion factor (ratio of spontaneous emission rate per mode to the stimulated emission rate per photon) and the linewidth enhancement factor. From a theoretical perspective, the spontaneous emission rate per mode can be readily calculated, and the presence of the inversion factor is unnecessary. A modification of the linewidth formula yields the following simple expression: ${ }^{16}$

$$
\delta \omega_{S T}=\frac{\beta R_{\mathrm{sp}}}{4 \pi S}\left(1+\alpha^{2}\right),
$$


where $R_{\mathrm{sp}}$ is the total spontaneous emission rate, $\beta$ the fraction of spontaneous emission coupled into the lasing mode, $S$ the photon density in the lasing mode, and

$$
\alpha=\frac{d \chi_{R}(n) / d n}{d \chi_{I}(n) / d n},
$$

where the real and imaginary parts of the differential susceptibilities are functions of the carrier density and given by: ${ }^{4,6}$

$$
\begin{aligned}
& \frac{d \chi_{I}(n)}{d n}=\int d E \frac{d g(n, E)}{d n} \frac{\hbar / T_{2}}{\left(E-E_{1}\right)^{2}+\left(\hbar / T_{2}\right)^{2}}, \\
& \frac{d \chi_{R}(n)}{d n}=\int d E \frac{d g(n, E)}{d n} \frac{E-E_{1}}{\left(E-E_{1}\right)^{2}+\left(\hbar / T_{2}\right)^{2}},
\end{aligned}
$$

where $d g / d n$ is the differential gain, $E_{1}$ the lasing energy, and $T_{2}$ the collisional broadening time due to carrier-carrier and carrier-phonon interactions, taken to be 0.2 ps. If $T_{2} \rightarrow \infty$, Eq. (3) contains a delta function, and Eq. (4) is a representation of the fact that $\chi_{R}$ is the Kramers-Kronig (Hilbert) transform of $\chi_{I}$.

This formulation of the Schawlow-Townes expression does not usually represent significant progress since one parameter $n_{\mathrm{sp}}$ is replaced by another, $\beta$. The latter is difficult to compute exactly in a macroscopic cavity laser, primarily owing to its small magnitude. In strongly index-guided lasers, $\beta$ has been estimated ${ }^{16}$ to equal $10^{-5}$. Nevertheless, $\beta$ is expected to be approximately inversely proportional to the cavity length since the number of allowed longitudinal modes is doubled when the cavity length is doubled. By an extension of the direct calculations of the electronic band structure in the strained quantum well active region ${ }^{17}$ and of the photon density of states ${ }^{13-15}$ in the surface-emitting microcavity, it is possible, however, to determine $\beta$ accurately. ${ }^{14,15}$ The total spontaneous emission rate is given by (in cgs units):

$$
\begin{aligned}
R_{\mathrm{sp}}= & \int d(\hbar \omega) \frac{4 \pi^{2} e^{2} \hbar}{\eta^{2} m_{0}^{2} \hbar \omega} \frac{1}{2 \pi^{2}} \sum_{n, m} \int d K \sum_{\sigma, \hat{\epsilon}}\left|\hat{\epsilon} \cdot \mathbf{P}_{n m}^{\sigma}(k)\right|^{2} \\
& \times \rho_{e}(\hbar \omega) \delta\left[E_{n}^{e}(k)-E_{m}^{h}(k)-\hbar \omega\right]\left\{f^{e}\left[E_{n}^{e}(k)\right]\right\} \\
& \times\left\{1-f^{h}\left[E_{m}^{h}(k)\right]\right\},
\end{aligned}
$$

where $P_{n m}(k)$ is the momentum matrix element between the $n$th subband in the conduction band and the $m$ th subband in the valence band, $f^{e}$ and $f^{h}$ the electron and hole distribution functions, respectively, $\rho_{\hat{\epsilon}}$ the density of states for polarization $\hat{\epsilon}, \hbar \omega$ the photon energy, $k$ the electron wave vector in the plane of the quantum well, $E^{e}$ and $E^{h}$ the electron and hole energies, respectively, in the indicated subbands, $\eta$ the refractive index in the active layer, $m_{0}$ the free-electron mass, $e$ the electron charge, and $\hbar$ the reduced Planck constant. The method and quantitative results for the calculations of the photon density of states from solutions of the wave equation in the surface-emitting microcavity have been presented elsewhere. ${ }^{14,15}$ In a brief summary, the wave equation is separated in the transverse and longitudinal coordinates, with the solutions for the former computed analytically for a square cross section of the surface-emitting laser cavity. All of the transverse modes are assumed to be discrete and correspond to discrete eigenvalues owing to the total internal reflection at the boundary between the semiconductor and air. ${ }^{13}$ The solutions in the longitudinal direction are obtained via a transfer-matrix approach separately for $s$ - and $p$-polarized modes. Three classes of modes are recovered: (i) resonant modes resulting from a quarter-wavelength phase slip in the center of the center of the cavity, (ii) leaky modes with wave vectors beyond the optical band gap induced by the quasi-periodic mirror stacks, and (iii) horizontally propagating modes similar to the usual longitudinal modes in the edge-emitting laser cavity. Only the first class of modes is capable of lasing owing to the large outcoupling losses associated with the other two classes. The modes of the full three-dimensional cavity are obtained as crosspoints of the longitudinal and transverse modes, the derivation of which is outlined above. The leaky modes are essentially unconfined by the cavity and contribute a normalized 1D density of states for each transverse eigenmode. The resonant and propagating modes contribute a discrete mode each for each transverse eigenvalue. A Lorentzian line shape is assumed for discrete modes with the linewidth determined by the reflectivity associated with each mode class. ${ }^{14,15}$ The calculation of the band structure and optical transition-matrix elements in strained quantum wells using the Kohn-Luttinger Hamiltonian has also been discussed at length. ${ }^{17}$ The spontaneous emission rate into a given mode can then be found by restricting the integral of Eq. (5) over the appropriate wave-vector spread, inversely related to the cavity quality factor. The latter is determined from the reflectivities for the propagating and confined modes as discussed extensively in Ref. 13. The linewidth enhancement factor can be readily computed for a given carrier density in the quantum well using Eqs. (2)-(4) and extending the numerical approach of Ref. 17. The laser rate equations ${ }^{16}$ can be used to find the output photon density versus the injected current density. The results for a microcavity surface-emitting laser with lateral widths of 0.5 and $1 \mu \mathrm{m}$ and a mirror reflectivity of 0.991 for normal incidence are shown in Fig. 1. For comparison, the light-current characteristic of a conventional surfaceemitting laser with a cavity width of $20 \mu \mathrm{m}$ is also displayed. The required value of the reflectivity can be achieved by a variety of designs, such as $\mathrm{Al}(\mathrm{Ga}) \mathrm{As} / \mathrm{GaAs}$ stacks or compact dielectric multilayer mirrors. All quantum well lasers have an active medium consisting of a single $50 \AA$ $\mathrm{In}_{0.2} \mathrm{Ga}_{0.8}$ As quantum well with built-in compressive strain on a GaAs substrate.

\section{DISCUSSION}

The derivation of Eq. (1) assumes that spontaneous emission is irreversible owing to the coupling to the thermal reservoirs of the modes of the universe and of the crystal lattice. The interaction of a two-level system at $0 \mathrm{~K}$ and of a single-mode field is, in principle, reversible with the continuous energy exchange between the field and the system (Rabi oscillations).$^{18}$ However, in practical microcavity lasers, the photon lifetime (a few ps) and the dipole lifetime (tenths of a ps) are much smaller than the period of Rabi oscillations (tens of ps) resulting in a loss of coherence. The dipole lifetime cannot be greatly increased by lowering the temperature due to the strong carrier-carrier interaction. From Eq. (1), 


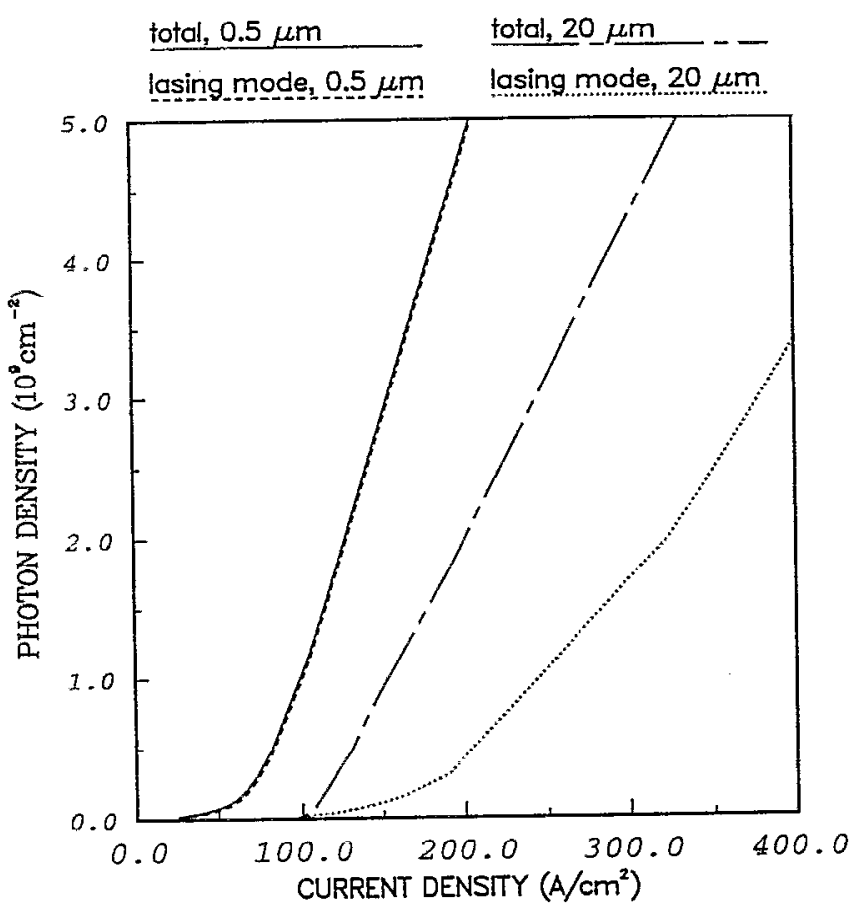

FIG. 1. The photon density vs the injected current density for the 0.5 and 20 $\mu \mathrm{m}$ wide cavities in all modes and in the lasing mode only.

the linewidth of a semiconductor laser is directly proportional to the rate at which spontaneous emissions perturb the phase of the optical field in the lasing mode. At higher field intensities (higher photon densities), the dephasing effect of quantum noise becomes less important. Since the rate of spontaneous emission into the lasing mode is greatly enhanced in a microcavity laser, it is natural to expect linewidth broadening by several orders of magnitude. For example, $\beta=0.1,0.05$ for the microcavity lasers with a cavity of width of 0.5 and $1.0 \mu \mathrm{m}$, respectively, compared with $\beta \approx 10^{-5}$ in the DFB laser. If the active regions in the DFB and microcavity lasers are identical, it might appear from Eq. (1) that the linewidth is proportional to the spontaneous emission coupling factor. Nevertheless, numerical simulations show that linewidth broadening is much slower than $\beta$ enhancement. For $\beta$ sufficiently close to unity, the total spontaneous emission rate is modified along with its distribution between the cavity modes. Owing to the insufficient filling of the wave-vector space by discrete transverse modes in the surface-emitting geometry, the total spontaneous emission rate may be reduced by a factor of $2-3$, a phenomenon also manifested in the suppression of the threshold current density. ${ }^{14}$ A combination of a microscopic cavity and a strained active layer also allows a slight reduction in the threshold current density with the concomitant reduction in the linewidth enhancement factor. The spectral linewidth versus the photon density is shown in Fig. 2 for two microcavity lasers, a DFB laser with a $100 \mathrm{~nm}$ thick active region and a strained quantum well active layer. The majority of experimental measurements of the linewidth to date have focused on the bulk DFB laser. The reported linewidths are in the 10-100 MHz range for output powers of several $\mathrm{mW}$. An

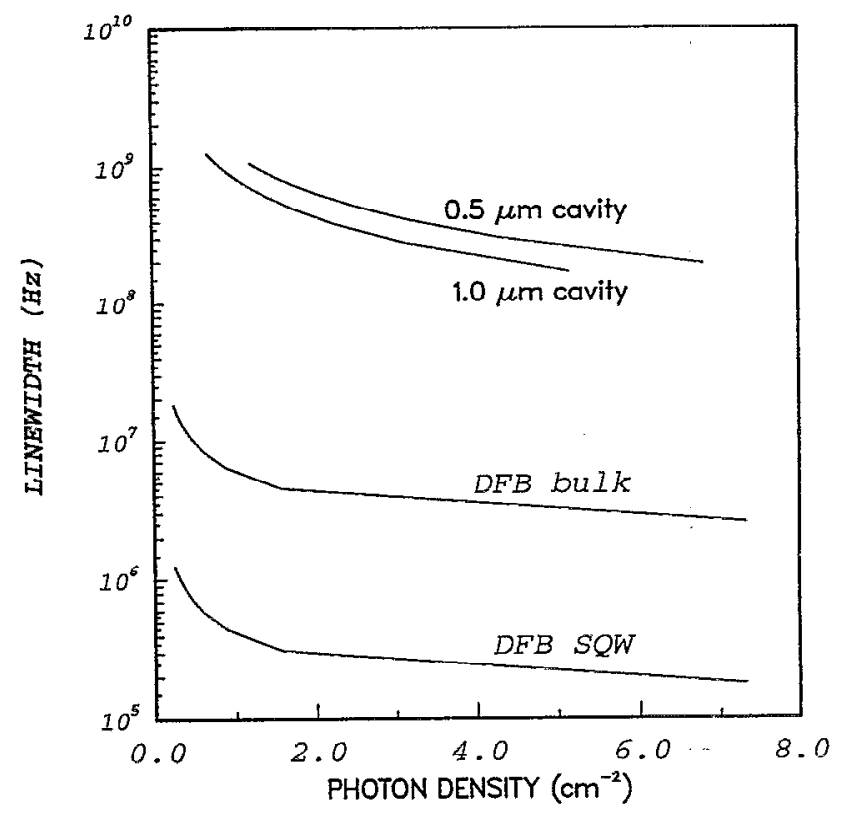

FIG. 2. The spectral linewidth vs the photon density in microcavity lasers with cavity widths of 0.5 and $1.0 \mu \mathrm{m}$, a bulk DFB laser with a $100 \mathrm{~nm}$ thick active layer, and a strained quantum well DFB laser.

order-of-magnitude reduction has been demonstrated in multiquantum well DFB lasers. ${ }^{19}$ By substituting typical values for the active area and quantum efficiency, the linewidth calculated in Fig. 2 for DFB lasers is smaller by a factor of $2-4$. The discrepancy may be attributed to (i) uncertainty in the $\beta$ factor, for which only an order-of-magnitude estimate is available, (ii) the neglect of the effects of gain saturation at high photon densities, and (iii) the neglect of the additional enhancement factors derived from nonorthogonality of transverse and longitudinal cavity modes. ${ }^{20}$ Note also that, on the basis of this model, the linewidth is expected to be approximately inversely proportional to the cavity length. The results of Fig. 2 also indicate almost an order-of-magnitude line narrowing in the strained quantum well laser as compared with the double heterostructure laser. The linewidth reduction is solely due to the smaller value of the linewidth enhancement factor.

The results of Fig. 2 demonstrate that a spectral linewidth of several hundred $\mathrm{MHz}$ is to be expected theoretically from surface-emitting microcavity lasers with strained quantum well active regions. Another useful comparison that can be made is in terms of the injected current density. The results of theoretical calculations are summarized in Fig. 3. Only the linewidth above threshold is shown since the use of the Schawlow-Townes formula below threshold requires some care. ${ }^{21}$ The relation between laser threshold and linewidth narrowing is discussed further below. The threshold current density in bulk DFB lasers is close to $1000 \mathrm{~A} / \mathrm{cm}^{2}$, while it is only $160 \mathrm{~A} / \mathrm{cm}^{2}$ in strained quantum well lasers. The threshold current density of the microcavity lasers is further reduced to $60-80 \mathrm{~A} / \mathrm{cm}^{2}$, depending on the lateral width. Therefore, as can be seen from Fig. 3 , the linewidth enhancement in microcavity lasers is further countervailed 


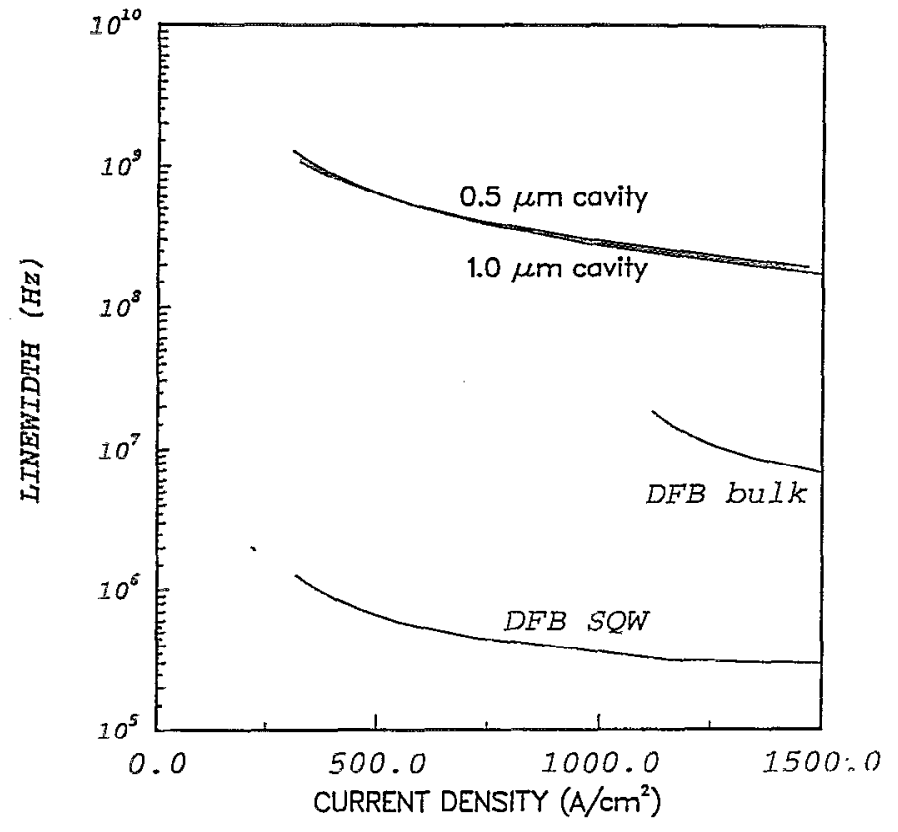

FIG. 3. The spectral linewidth vs the photon density in microcavity lasers with cavity widths of 0.5 and $1.0 \mu \mathrm{m}$, a bulk DFB laser with a $100 \mathrm{~nm}$ thick active layer, and a strained quantum well DFB laser.

by threshold reduction, i.e., the difference in the spectral linewidth is reduced if the comparison is made at the same current injection. The calculations made for the DFB laser assume that the laser oscillates in a single mode as long as it is biased above threshold. It must be recognized that not for all so-called single-mode lasers, is this assumption sound. On the other hand, properly designed microcavity lasers are intrinsically single mode both above and below the lasing threshold since only one resonant mode accepts a significant portion of spontaneous emission.

In a macroscopic cavity laser, the relation between the linewidth far below and far above lasing threshold is easily understood. In the former case, the linewidth is of the order of the "cold" cavity linewidth inversely related to the cavity quality factor. In the latter case, the linewidth is inversely proportional to the output optical power. In the vicinity of the lasing threshold, more complex behavior has been observed and analyzed. ${ }^{21}$ In microcavity lasers whose light-current characteristic is shown in Fig. 1, the lasing transition is less pronounced, but the general conclusions arrived at for macroscopic cavity lasers are still valid. In truly "thresholdless" microcavity lasers, for which $\beta$ must exceed $0.5,{ }^{15}$ the curvature in the light-current curve disappears, and the threshold current density defined as the intersection of the light-current curve with zero photon density is almost exactly zero. The question arises: Can a linewidth significantly narrower than the "cold" cavity linewidth be expected in microcavity lasers for any value of injected current? The answer can be obtained by recollecting that the Schawlow-Townes expression accounts for the linewidth due to quantum noise arising from spontaneous emission processes. If the optical field is treated classically, the laser emission line is a delta function if the gain of the active medium balances the reflection and intrinsic losses in the cavity. ${ }^{22}$ In a macroscopic cavity laser, the threshold current obtained by finding the current density intercept as discussed above is close to the point at which the gain-loss balance becomes nearly perfect. However, this is no longer true in a thresholdless microscopic cavity laser. A finite injection current is always necessary to achieve transparency in the active medium. Sufficiently far below that point, linewidths of the order of those in a "cold" cavity are to be expected.

\section{CONCLUSIONS}

To summarize, in this paper we have presented the results of our numerical calculations for the spectral linewidth in micrometer-sized microcavity surface-emitting lasers noting the reasons for the absence of the "catastrophic" linewidth broadening expected from spontaneous emission coupling factor considerations alone and given an intuitive discussion of the various factors that must be taken into account if an adequate understanding of the linewidth in macroscopic and microscopic cavity lasers is desired.

\section{ACKNOWLEDGMENT}

This work was supported by the Army Research Office under Contract No. DAAL 03-92-G-0109.

${ }^{1}$ M. W. Fleming and A. Mooradian, Appl. Phys. Lett. 38, 511 (1981); 40, 865 (1982).

${ }^{2}$ Originally presented by A. L. Schawlow and C. H. Townes, Phys. Rev. 112, 1940 (1958); corrected by M. Lax, in Physics of Quantum Electronics, edited by P. L. Kelley, B. Lax, and P. E. Tannenwald (McGraw-Hill, New York, 1966), p. 735; H. Haug and H. Haken, Z. Phys. 204, 262 (1967).

${ }^{3}$ C. H. Henry, IEEE J. Quantum Electron. QE-18, 259 (1982); QE-19, 1391 (1983).

${ }^{4}$ K. Vahala, L. C. Chiu, S. Margalit, and A. Yariv, Appl. Phys. Lett. 42, 631 (1983).

${ }^{5}$ C. Harder, K. Vahala, and A. Yariv, Appl. Phys. Lett. 42, 328 (1983).

${ }^{6}$ Y. Arakawa, K. Vahala, and A. Yariv, Appl. Phys. Lett. 45, 950 (1984); Y. Arakawa and A. Yariv, IEEE J. Quantum Electron. QE-21, 1666 (1985).

${ }^{7}$ W. Elsässer and E. O. Göbel, IEEE J. Quantum Electron. QE-21, 687 (1985); K. Vahala and A. Yariv, Appl. Phys. Lett. 43, 140 (1983).

${ }^{8}$ W. Elsässer, Appl. Phys. Lett. 44, 1126 (1984).

${ }^{9}$ F. Herzel, K. Henneberger, and W. Vogel, IEEE J. Ouantum Electron. QE-29, 2891 (1993).

${ }^{10}$ G. P. Agrawal, IEEE J. Quantum Electron. QE-23, 860 (1987); J. Appl. Phys. 63, 1232 (1988).

${ }^{11}$ G. P. Agrawal, IEEE J. Quantum Electron. QE-26, 1901 (1990).

${ }^{12}$ Y. Yamamoto, S. Machida, and G. Bjork, Opt. Quantum Electron. 24, S215 (1992); H. Yokoyama and S. D. Brorson, J. Appl. Phys. 66, 4801 (1989).

${ }^{13}$ T. Baba, T. Hamano, F. Koyama, and K. Iga, IEEE J. Quantum Electron. QE-27, 1347 (1991); QE-28, 1310 (1992).

${ }^{14}$ I. Vurgaftman and J. Singh, Appl. Phys. Lett. 64, 1472 (1994).

${ }^{15}$ I. Vurgaftman and J. Singh (unpublished).

${ }^{16}$ G. P. Agrawal and N. K. Dutta, Semiconductor Lasers, 2nd ed. (Van Nostrand Reinhold, New York, 1993).

${ }^{17} \mathrm{~J}$. P. Loehr and J. Singh, IEEE J. Quantum Electron. QE-27, 708 (1991).

${ }^{18}$ M. Sargent III, M. O. Scully, and W. E. Lamb, Jr., Laser Physics (Addison-Wesley, London, 1974).

${ }^{19}$ K. Uomi, S. Sasaki, T. Tsuchiya, M. Okai, M. Aoki, and N. Chinone, Electron. Lett. 26, 52 (1990).

${ }^{20}$ W. A. Hamel and J. P. Woerdman, Phys. Rev. A 40, 2785 (1989); Phys. Rev. Lett. 64, 1506 (1990).

${ }^{21}$ R. Hui, N. Caponio, S. Benedetto, and I. Montrosset, IEEE Photon. Technol. Lett. 4, 841 (1992).

${ }^{22}$ For a "philosophical" discussion of this point, see R. Lang, M. O. Scully, and W. E. Lamb, Jr., Phys. Rev. A 7, 1788 (1973). 